\title{
Standardization of Testing the Level of Physical Fitness of Students of 18 - 20 years
}

\author{
Nadezhda I. Palagina \\ Michael M. Polevshchikov \\ Yulia A. Dorogova \\ Natalya V. Familnikova \\ Mari State University, Yoshkar-Ola, Russia \\ Email: palaginanadiv@yandex.ru \\ Maria L. Blinova
}

Mari State University, Yoshkar-Ola, Russia, Interregional Open Social Institute, Yoshkar-Ola, Russia,

Email: airam8@mail.ru

\section{Doi:10.5901/mjss.2015.v6n3s7p265}

\begin{abstract}
In this article there is considered the problem of a standardization of testing the level of physical fitness of girls students. At current the method of testing is one of the main methods of pedagogical control of the level of physical fitness of exercisers'. In this case, the tests are the motor task, the results of which performance is evaluated the level of development of motor skills that form the basis of physical fitness person. One of the criteria that determine the accuracy of the estimate is the coefficient of reliability of the proposed test. It was found that the coefficient of reliability of motor tests can be improved by standardizing testing. Standardization is the rules and procedures of the various tests and associated actions. The article presents a method of testing for the five motor tests that are part of a comprehensive test battery to assess the level of physical fitness of girls students who not involved in sports: jogging 10 minutes; flexion and extension arms in emphasis lying on the lap in 30 seconds; raise the trunk in 30 seconds from a prone position on his back, arms at your sides, feet are not fixed ( hands-free ); "dislocate" with straight arms back with gymnastic stick; bending forward, standing on a gymnastic bench. The article also discusses the methodology of mass testing of 954 students in accordance with the requirements of standardization.
\end{abstract}

Keywords: standardization of testing, safety factor, physical fitness, testing procedure.

\section{Introduction}

Physical fitness is the result of human physical training, which ultimately aims to strengthen the organs and systems of the organism, to improve the functional abilities for the development of motor qualities (strength, speed, endurance, flexibility, agility) (Ozolin, 1988). Physical training is divided into general and special (Boyko, 1976; Viktorov, 1990; Matveev, 1991; Palagina, 2005). Special physical training is aimed mainly at strengthening the organs and systems, the increase their functional abilities, the development of motor qualities strictly in relation to the requirements of the chosen sport. At the training sessions with the students of the basic and preparatory education departments (not involved in sports) are mostly used the means and methods of general physical training. Under the influence of the general physical training the health of students improves, increases efficiency, it becomes a perfect body, he adapts quickly to stress and reach a higher level in the development of motor skills. In the physical training of students, there are three areas:

- - -development of all organs and systems;

- $\quad$-development, strengthening and improving the individual units of the body;

- - -strengthening the "weaknesses" in the body, increasing the functionality of the "laggards" organs and systems (Palagin, Palagina, Polevshchikov, 2008; Palagina, Polevshchikov, 2013; Palagina, Polevshchikov, 2014; Palagina, Polevshchikov, Dorogova, Blinova, Zakamsky, Shraga, Loskutova, 2015).

The effectiveness of the process of physical training of a human performed by assessment of the level of physical fitness of exercises, or rather its motor abilities. It should be noted that of all the traditional existing methods of the pedagogical control (collection of opinions, analysis, monitoring, measuring) the current method of testing is the main 
method of evaluation and control of the level of physical fitness of students, due to its motor abilities (Ostanigrosh, 1984; Palagina, 2005; Palagina, Blinova, 2010; Polevshchikov, Palagina, Schraga, 2010).

Motor abilities of the person combined in whole the concept of "movements", based, as Y.I. Smirnov, in a series of neuro-endocrine and muscle metabolic factors causing displacement in the space of the human body and its segments (Smirnov, $1989 \mathrm{a}, \mathrm{b}$ ).

The sports- pedagogical sense "movements" means a motor skills of a human, determined by its achievements in the various physical exercises - tests called abroad «motor fitness test» (Buba, 1968).

Motorial tests are motor tasks, allowing to determine the state of human motor functions: a) the level of physical qualities; b) the ability to perform a certain range of motion (motor abilities, skills) (Palagina, 2005; Palagina, Polevshchikov, Dorogova, Blinova, Zakamsky, Shraga, Loskutova, 2015).

The majority of national and foreign authors advocate a comprehensive approach to assessing the physical fitness of the person disagree about the quantity and quality of the necessary indicators (Palagina, 2006). At different times by different authors for the assessment of physical fitness it was used by one - two to fifteen or more test parameters (Hasin; Rafalovich, 1995). In drawing up the test systems for the identification and assessment of physical fitness of students this problem is solved ambiguously in various countries. Analysis of the literature showed that in abroad and in our country, in the modern theory and practice to assess the physical fitness of a person use a plurality of test systems. They differ from each other in the number and content of exercises within them (Palagina, 2005).

Many national and foreign scholars (Palagina, 2005) to assess the level of physical fitness of students use motor tasks that are most often used in practice (running, jumping, throwing). Another part of researchers, as E.Y. Bondarevskiy, under the selection of exercisesto the test combinations thinkthat the concept of physical fitness is necessary to include the requirements to possess certain skills (Bondarevskiy, Hankeldiev, 1986). Part of experts to assess the physical fitness of students prefer to use tests that assess endurance, strength, speed and agility and coordination in various forms of expression. It creates a huge number of test systems for the evaluation of physical fitness of students. One of the factors, which considered in the preparation of these complexes is the presence of indicators of safety factors used of motor tests. Thus the preference (with a small difference in the coefficients of factor) was given to the tests are already widely used in practical, easy to use and requires no special equipment (Palagina, 2005). These various studies on the value of the coefficient of reliability (Bondarevskiy, Hankeldiev, 1986) indicate the absence of consensus on this issue, allowing for arbitrary use of a wide range of motor tasks even with the low value of the coefficient of reliability.

It should be noted that this approach to the formation of test combinations provides a qualitative description of the level of physical fitness and a more accurate assessment of motor abilities of students.

All this shows the complexity and the possibility of alternative approaches to resolving problems of formation of complex tests to assess physical fitness of young people. Differences of opinion of experts on how to be a test of physical fitness leads to a large number of tests to assess motor abilities. However, the problem of testing and evaluation of physical fitness person and students has not received a single solution up to date.

The problem of reliability of the test is always the center of attention of researchers. Under the reliability test means the degree of coincidence with results of the second test of the same testee under the same conditions (Bondarevskiy, Hankeldiev, 1986; Godik, 1988; Zatsiorsky, 1979; Palagina, Polevshchikov, Dorogova, Blinova, Zakamsky, Shraga, Loskutova, 2015; Smirnov, 1989 a, b). We differentiate between the stability of the dough, the consistency and equivalence. Equivalence tests determined if one and the same motor characteristics can be measured by several tests. If the results of the evaluations are the same, then it shows the equivalence of tests (Smirnov, 1989b). The coefficient of equivalence is determined by correlation coefficient calculated between the results of testing. Stability of the test depends on the type of test, on the contingent of the testee and on the time interval between the test and retest (Bondarevskiy, Hankeldiev, 1986; Zatsiorsky, 1979; Smirnov, 1989b). It is assessed the stability of the test or by using analysis of variance or by the coefficient of correlation between the test and retest (Zatsiorsky, 1979). Consistency test is characterized by independence of test results on the personal qualities of the person who carried out the test or evaluate (Zatsiorsky, 1979). This property is depend on the coincidence of testing methodologies for different specialists (Smirnov, 1989b).

Considering the question about the reliability of motor tests, it is necessary to pay attention to the fact that the reliability of the test can be improved to a certain extent by the strict testing standards (Palagina, Polevshchikov, 2003; Smirnov, 1989b).

According to leading experts in the field of metrology sportsa standardization of tests and measurements in these results is an important requirement in the testing of motor abilities, because it affects the accuracy of the testing results. Otherwise the differences in results under running may be a consequence of the fact that in the first test subjects ran into 
the arena with a 200- meter track, and in the second - in the stadium (Godik, 1988; Palagina, Polevshchikov, Dorogova, Blinova, Zakamsky, Shraga, Loskutova, 2015).

Standardization makes special demands on the accuracy, reliability and objectivity of the collection and processing of the scientific data and test the validity of their application in each particular case. The aim of standardization is to achieve the optimum degree of order established by the provisions, requirements and standards for solving real-life problems (Palagina, Polevshchikov, 2003; Smirnov, 1989b).

The standardization process produced rules, regulations, requirements, specifications relating to the object of standardization, which are issued in the form of a regulatory document. Among other standards, considerable importance is attached to the standard test methods that sets the rules and procedures of the various tests and associated actions (Palagina, Polevshchikov, 2003; Smirnov, 1989b).

Based on their own researches and observations, L.A. Hasin and A.B. Rafalovich noted that the absence of strict standardization exercises that assess the mobility of certain joints, coordination, agility and balance the body, contributes to variation in the performance of these exercises, and as a result reduces the reliability of the test (Hasin; Rafalovich, 1995).

In this study "Comprehensive assessment and control of sports readiness" Y.I. Smirnov noted that testing is necessary to achieve the unity of determination and the establishment of requirements to them, the lack of which generates most of the errors in the obtained results (Smirnov, 1989b). In order to ensure uniformity of testing procedures, it is necessary to make a program and testing methodology. The program should reflect the object, purpose, scope and content of tests. The methodology should establish the ways to conduct tests to determine the parameters or parameters of physical fitness of each property listed in the program.

According to experts (Smirnov, 1989b), the effectiveness of the testing program determined the following characteristics:

1) test parameters apply to this type of activity (or sports);

2) selected tests are simultaneously reliable and valid, ie the test is justified when actually determines what is to be determined, and is dependable when test results are logical and reproducible;

3) testing protocols are the most specific to a particular activity (or sports specialization), and that the results of the tests were the most practical value, type of loads should reflect the specifics of the activity (or sport);

4) the test requires a strict control; selected components of the testing should be carried out in sequence, which inevitably entails the standardization recommendations for artists and managers;

5) respect for human rights of the testee; ethical criteria (which must be followed before testing) include a detailed explanation of the purpose of tests, information about the possible physical or mental risks associated with their conduct and safety measures;

6) regular repetition of the test; since one of the objectives of testing is to monitor the efficiency of training, testing should be repeated after its various stages; a one-time test (one per year), although it has potential interest, has little practical value for the exerciser;

7) explanation of the test results - is the final stage; test results should be reported directly to the subject with an explanation understandable for him; then it is necessary to change a training program or to develop new ones based on this information.

According to the theory test (Godik, Shanin, Shitikova, 1973; Palagina, 2005; Smirnov, Polevshchikov, 2000), the standard of testing ensures compliance with a number of rules:

- day regimen preceding the test should be based on the same scheme;

- workout should be standard (in duration, exercise selection and sequence of their implementation);

- Testing should be carried out, if possible, the same people who can;

- diagram of the test should not be changed;

- intervals between the attempts of the same test should eliminate a fatigue arising after the first attempt;

- testee must strive to show the best results.

As V.M. Zatsiorsky (Zatsiorsky, 1979) notes, standardization means ordering the test conditions and procedures, which must be the same in all cases of the test. In the studied literature the detailed description of test methods is very rare. Most often, authors or indicate the name of the test and what it measures (or estimates), or list a motor task included in the test of physical fitness (Palagina, 2005). Some sources (Palagina, 2005) give a partial description of the proposed tests, but there is no test program.

The undeniable interest and certain value are recommendations which are given in article of V.I. Lyakh (Lyakh, 1987) to ensure adequate levels of motivation and concentration of the testees for the forthcoming activities, so they can show off their best results: 
Firstly, the test should be clearly informed about the purpose of control tests;

Secondly, it should to explain in detail, to demonstrate, and then to teach them to perform the tests;

Thirdly, the test should be performed at the beginning of the main part of the lesson after the introduction, which promotes a functional training of the body dealing with the upcoming activities.

The role of the warm-up, as a preparatory stage in order to achieve maximum results in the subjects tested, confirmed by many studies, as detailed in the thesis of I. A. Vardishvili (Vardishvili, 1954). Speaking about the nature of a warm-up, he noted that more effective impact on most of the work have such preliminary exercises that mimic the basic movements, both in structure and in the rate, but on the condition that the interval between the preliminary and the main work does not exceed 3 - 5 minutes. Besides, as the author notes, the warm-up has an effect on the state of the higher parts of the central nervous system, in their excitability, and thus the occurrence of emotional state gives a burst of energy, a kind of ready for use. It was established that for the preparation of the body for the upcoming test activities it is enough 5 - 10 minutes of a warm-up (Vardishvili, 1954).

In the available literature the tests meeting the requirements of standardization, often presented in a foreign sources (Palagina, 2005). Recently, andinthe Russian literature ("Presidential tests", etc.) (Palagina, 2005) there is a tendency of standardization of tests and testing methods, which significantly improves the quality of a control of the level of development of motor abilities of students and makes it possible to compare test results of young people of various educational institutions.

\section{Methods, Approaches}

In order to standardize test the level of physical fitness of students, and to obtain objective data characterizing the full physical fitness of students in universities of Yoshkar-Ola was organized a survey of students of 1 - 3 courses of different faculties (Palagina, 2005). As a result, the study involved more than 900 students who are not involved in sports. For this it were used the tests, describing the different sides of physical fitness:

1. Running for 10 minutes imposes certain requirements for aerobic capacity of the organism to the state of cardio-respiratory and hormonal systems in the muscles of the legs, back and abdomen.

2. Flexion and extension arms in emphasis lying on the lap in 30 seconds - the results characterize the work of power-speed mode, muscles in the arms, shoulder girdle.

3. Raising the trunk in 30 seconds from a prone position on his back, arms at your sides, feet are not fixed (hands-free) - characterize the results of work in the power-speed mode, the abdominal muscles.

4. "Dislocate" by straight arms back with gymnastic stick - on the results of this test gives a comprehensive description of the mobility in the shoulder joint.

5. Tilt forward, standing on a gymnastic bench - the results can be seen on the slope of mobility in the hip joints, as well as the flexibility of the spine, the elasticity and extensibility of the ligaments and the muscles involved in performing this test.

Based on the fact that the flexibility in the greatest part due to elongation of musculo- ligamentous apparatus, and were selected such exercises, under which the movement would be committed in the most important joints of the musculoskeletal system, and subjected to stretch the largest muscle groups of the body. Besides, in the practice of physical training the most important is the mobility in the joints of the spine, hip joint and shoulder joint zone, the amplitude of movements in which during the life tends to decrease.

According to researchers ((Vasiliev, 1965; Matveev, 1991; Palagina, 2005) the slope of the trunk forward while standing on straightened legs, can give a comprehensive description of the level of overall flexibility. In favor of the exercise by the following circumstances:

firstly, when the forward tilting movement occurring in not one but in several, and moreover the largest and most important joints of the human body; range of motion in these joints during life tends to decrease;

secondly, this exercise describes the elongation of the largest muscles of the human body - back and hip extensors;

thirdly, the slope of the trunk forward is a simple exercise of its coordination structure, requires no prior training and is available to people of different age, sex and physical fitness;

fourthly, the amount of tilt easily and with sufficient accuracy can be determined by simple measurement devices under normal conditions of physical training; and the measurement results are expressed by objective numerical targets and visibility to the subjects themselves;

fifthly, the systematic use of forward tilt enables to carry out regular monitoring of the progress of the development of flexibility to properly assess the appropriateness of the means and methods. 
Control and teacher testing was at the mass testing of physical fitness of students who not involved in sports, using the generated test pattern. It was conducted on the basis of the three universities of Yoshkar-Ola.

\section{Core Results}

In the first phase of standardization was developed testing methodology for each motor tests included in the test battery. Have been developed to fulfill the requirements of tests (standardized tests) (Palagina, 2005).

Test 1. Running 10 minutes, held at the stadium, in the 400 - meter track with a layout of 100 meters. Testees themselves counting the distance they run for 10 minutes, up to $100 \mathrm{~m}$.

There is another version of the 10-minute run, for example, in the hall (the equivalence ratio was 0,93 ), but it is necessary to know the yardage on the perimeter of the room, which you will be running. It should be remembered that subsequent testing in the race for 10 minutes should be carried out in the same place where the previous one. Otherwise, the results are unreliable.

Notice. This test can be performed in conjunction with walking. In this case, difficulty performing continuous running the testee can go a step before the restoration of breathing, and then go back to running. Counted all the footage, and the one that ran through the student, and one that she has passed.

Test 2. Lifting body in 30 seconds is as follows. Starting position - lying on his back (on a mat or rug), legs straight, are not fixed, the hands are on the floor at your sides, palms pressed to the floor. On a signal (both included stopwatch) testee performed lifting the torso, coming to the position: saddle, hands forward. At the same time she was not allowed to help herself (based on his elbows, waddle side to side, "rip " feet on the floor (mat), stick to the legs (thighs)), or raise the body is not counted. It is necessary always to go back to the starting position, putting his hands on the floor, or the subsequent lifting of the trunk is also not counted. When performing this test, the number of correctly executed lifting the torso is registered, which the subject had time to run for 30 seconds.

Notice. You can allow a second attempt at the request of the testee after the recovery rate, but, as experience has shown, it does not give the desired result.

Test 3. Forward bend is performed on a method described by N.G. Ozolin and M.S. Abramov (Palagina, 2005). The measurement of results by using a simple device - a centimeter ruler, which is attached to the site of the gymnastic bench so that the zero point should be at the level of the surface of the bench. The testee stands on a bench so that the exposed end of the line is between the toes. Wherein: the foots are parallel to each other, and the toes do not protrude beyond the edge of the site. Testee slowly (do not perform springy movements) performs leaned forward to the limit, touching fingers line without bending your knees and hold the pose for three seconds. The measurement is made from the edge of the bench (zero centimeter mark on the ruler) to the third finger of both hands on the centimeter range. If the fingers do not reach the edge of the bench, the result is recorded in the minutes with a minus sign (-) if fall below - the plus sign (+).

Notice. The testee allowed to perform three attempts, the best result is entered on record. It is necessary for the testees to pay attention to the fact that evaluation of the flexibility made by the middle fingers of both hands, rather than one, i.e. fingers should be parallel to each other.

Test 4. Flexion and extension of the hands on the knees-ups in 30 seconds. Starting position - the testee takes abutment lying on the floor, and then, bending her knees, kneels. Thus, the legs and feet are in the top position and the shoulders, back and hips (knees) draw up one line. Under the arm bending the testee should try to touch the breast of the floor (thus it is impossible to bend back or arch the back), and push-ups when fully straighten arms. When there is a violation of these conditions press-up is not counted. The protocol (or register) entered the number of correctly performed press-ups, which the testee had time to run for 30 second.

Notice. At pleasure, a girl can perform a second attempt after recovery rate.

Test 5. "Dislocate" with straight arms back with gymnastic stick. To perform the job on this test, you must have a gymnastic stick and measuring tape. Firstly, the width of the shoulders is measured, then the testee takes gymnastic stick grip on top of the shoulder width, trying to move her arms straight above his head behind his back. If you can not to make "dislocate" grip at shoulder width, the hands sliding over the surface of the stick under testee increases the width of the grip to the point yet not be able to make "dislocate" with straight arms and return to starting position.

There are two ways to perform this test:

1) gymnastic stick is taken by shoulder-width grip on the right (or left) end and slide the left (or right) hands over the surface of the stick to increase the width of the grip;

2) gymnastic stick is taken by shoulder-width grip on the middle and slid both hands to the sides of the surface increases the width of the stick grip. 
The calculation of a result is as follows. Measuring tape measure the width of the grip (the distance between the wrists on a stick), at which managed to perform well "dislocate". This result (width grip) subtracted result obtained by measuring the width of the shoulders. The difference enters on record. The result (the difference between the width and shoulder width grip) and will be a measure of the mobility in the shoulder joint.

In carrying out this test, it is possible to allow two or three attempts (optionally test). In this case, the best result (smallest difference) enters on record (or log).

Notice. When performing this test should be careful. Firstly, you need a good workout on the muscles of the shoulder girdle flexibility, extensibility, as well as on the mobility of the shoulder joint. Secondly, to warn the subjects of pain in the shoulder joints. These feelings should be minor. It is not necessary to encourage girls who trying to show the best result, while experiencing severe pain that can lead to injury to the muscles, ligaments and tendons.

It is important to pay attention to the method of measuring the width of the shoulder: the measurement is taken from the exposed edge of the shoulder joint of the seventh cervical vertebra to the other protruding edge of the shoulder joint. Standardness of carry out the proposed test and measurement results are allowed to provide a high degree of reliability of the tests. Table 1 shows the performance of safety factors used motor tests.

Table 1 - Indicators of safety factors used motor tests (Palagina, 2005).

\begin{tabular}{|c|l|c|}
\hline №/n & Tests & Safetycoefficient \\
\hline 1. & Running 10 minutes & 0,900 \\
\hline 2. & $\begin{array}{l}\text { Raising the trunk in 30 seconds from a prone position on his back, arms at your sides, palms pressed to the } \\
\text { floor, his legs are not fixed }\end{array}$ & 0,904 \\
\hline 3. & Tilt forward, standing on a gymnastic bench & 0,975 \\
\hline 4. & Press-up in 30 seconds from the front plank on your knee & 0,951 \\
\hline 5. & "Dislocate" with straight arms back with gymnastic stick & 0,978 \\
\hline
\end{tabular}

\section{Consideration}

The coefficient of reliability, acceptable to assess the level of physical fitness is discussed since 1927 (Bondarevskiy, Hankeldiev, 1986). According to various studies (Palagina, 2005), it is in the range of 0,50-0,98. Many experts believe that the mass survey of motor abilities of students not involved in sports, suitable safety factor of at least 0,70 . Some experts argue that most of the tests in physical education should have a safety factor, which lies within a range from 0,90 to 0,99 (Palagina, 2005). The national sport of metrology specialists offer to evaluate the reliability of motor tests at five levels shown in Table 2 (Smirnov, Polevshchikov, 2000).

Table 2 - Gradation of levels of reliability tests used to assess the level of physical fitness

\begin{tabular}{|l|c|}
\hline Levels of safety factor of the tests of physical fitness & Interval \\
\hline 1. Low & $0,60-0,69$ \\
\hline 2. Acceptable & $0,70-0,79$ \\
\hline 3. Medium & $0,80-0,89$ \\
\hline 4. Fine & $0,90-0,94$ \\
\hline 5. Excellent & $0,95-0,99$ \\
\hline
\end{tabular}

Developed standardized of motor tests offered to achieve the following levels of safety factor of physical fitness tests in accordance with Table 2: running 10 minutes- 0,900 - good level; raising the trunk in 30 seconds from a prone position on his back, arms at your sides, palms pressed to the floor, his legs are not fixed-0,904-good level; bending forward, standing on a gymnastic bench - 0,975 - excellent level; press-up in 30 seconds from the "emphasis lying on his knees"0,951 - excellent level; "dislocate" with straight arms back with gymnastic stick - 0,978 - excellent level.

The mass testing was attended by testing 954 students of 1-3 courses of three high schools in Yoshkar-Ola. Experimenters prepared from among teachers of physical culture. The technique of mass testing included all of the recommendations developed in the process of standardization tests used to assess the level of physical fitness of students not involved in sports:

10 - minute run was carried out at the stadium on a treadmill $400 \mathrm{~m}$. Warm-up before the start included a variety of flapping arms, legs, torso twists to the side and exercises for the muscles of the foot. It was given a mass start. At the 
same time the experimenter activate a stopwatch. After 10 minutes sounded the last post discussed in advance with students. Girls independently calculated the distance they ran for 10 minutes up to $100 \mathrm{~m}$. The second and third attempts were repeated after 2-3 days for subsequent sessions. The results of all three attempts were entered on record, and then calculates the average result.

Raising the trunk in 30 seconds from a prone position on his back, arms at your sides, palms pressed to the floor, his legs are not fixed as follows. After an easy warm-up it was given the instructions on the correct exercises. Then for girls it was initiated a command to count off. Students under the number "one" occupy their original position on the mat or on soft floor coverings. Students under the number "two" are at the feet of the "first" and acted as "counters". After that "the first" did the job the students swapped the roles. After 30 seconds, it gives the command "Stop!" and students "meters" called the number of correctly executed tasks (lifting the torso). The result was entered on record. Then the girls changed their places, and the whole procedure was repeated. After restoring the pulse the students performed the second and then a third attempts. The results of all attempts were entered on record, which are calculated on the basis of the average.

Deep slope performed after the warm-up of stretching the major muscle groups involved in the implementation of this test. The testee performed three attempts in a row and all the results were entered on record, and then the average value was calculated.

Flexion and extension of the hands on the knees-ups in 30 seconds (press-up) also performed after a little warm up the overall impact.

As well during the test "lifting body", the girls were counted off. The "first" took their initial position on the flooring (mat), and the "second" rose from the head of the "first", acting as a "counter".

At the command of the experimenter (at the same time start the stopwatch) "first" performed push-ups, and the "second" counted the number of correctly performed press-ups in 30 seconds. After 30 seconds, it was a command "Stop!" and "counters" reported the number of correctly performed flexion and extension arms (press-ups) in the kneeling hips extended. The result was entered on record. Girls were reversed, and the whole process was repeated again. After restoring the pulse performs a second and then a third attempt. The results are entered on record, and then calculates an average value.

"Dislocate" with straight arms back with a gymnastic stick. Prior to testing, the girls performed exercises on major muscle groups of the shoulder girdle and hands to prepare them for the work ahead. Each of the testees performed three attempts in a row, each time starting from the grip width equal to the width of the shoulders. After each attempt the difference will be recorded, and then the average value was calculated.

Testing was conducted in the morning and before each test motor tasks sure to warm up. It was a set of preliminary exercise, contributing to the preparation of major muscle systems and systems for the upcoming work. This warm-up, according to some experts (Palagina, 2005), provides a faster "occurrence" of a person in work and contributes to the achievement of maximum results for each dealing. It is shown that a more effective impact on most of the work have such exercises that mimic its movements both in structure and in the rate of (Vardishvili, 1954). The scientists found (Palagina, 2005) the beneficial effects of the workout process "warming up" (when various systems and organs reach their optimal level of activity) for a long flowing muscle activity, as well as power and speed-power. Pre-stretching exercises increase muscle elasticity and extensibility of defining the mobility of joints, providing the necessary amplitude of their movement. It also noted that the pre-exercise has a positive effect on the subsequent basic work only when the interval between the preliminary and main work is not more than 3-5 minutes. Warm-up has an effect on the state of the higher parts of the central nervous system, on its excitability. The appearance in this emotional state gives a burst of energy, a kind of ready for use (Vardishvili, 1954).

In order to obtain objective data testing is always carried out by two teachers. Pre-briefing was held with them by the method of testing. To eliminate the subjectivity of assessment method was used retesting. Particular attention was paid to compliance with the standard in the performance test of motor tasks. It is possible to obtain high coefficients of reliability.

\section{Conclusions}

Results of the study on standardization of testing the level of physical fitness of girls not involved in sports, the following conclusions:

1. The effectiveness of the process of physical training performed by human assessment of the level of physical fitness involved, or rather its motor abilities. Standardization of testing and measurement in these results is an important requirement in the testing of motor abilities, because it affects the accuracy of the testing results. 
2. Standardized testing ensures compliance with recommendations to be followed for diagnostic (first) testing and control (again). These recommendations relate to:

- $\quad$ the day regimen preceding the test (it should be based on a standard scheme);

- a warm-up, which should be a standard (in terms of duration, exercise selection and sequence of their implementation);

- people conducting testing (testing should be carried out, if possible, the same professionally trained specialists);

- the test circuit (not to be changed);

- rest interval between attempts of the same test (rest intervals in duration must correspond to the period of liquidation of a fatigue arising after the previous attempt);

- testees who must strive to show their best results.

3. Standardization of testing the level of physical fitness of girls 18-20 years old students of Joshkar-Ola was caused by developed testing methodology for each motor tests included in the test battery, which in turn has provided a high degree of reliability of the tests. These tests can be used to assess the level of physical fitness of girls 18-20 years old, who are not involved in sports.

\section{References}

Bondarevskiy, E.Y., Hankeldiev, Sh. Kh. (1986). Physical fitness of students. Tashkent: Methodology 208.

Boyko, L.F. (1976). Basics of athletics. M.: Physical Education and Sports.

Buba, H. (1968). Tests in sports practice. M.: Physical Education and Sports, 98-130.

Vardishvili, I.A. (1954). About cortical regulation of cardiac activity and respiration during exercise warm-up question in sports: Diss .... Cand. biol. Sciences. Tbilisi, 100-121.

Vasiliev, E.P. (1965). Research body flexibility and experimental study of means and methods of its education:Diss .... Cand. ped. Sciences. Riga.

Viktorov, F.V. (1990) A method for rapid control over the level of the physical condition of the person. Journal of Theory and Practice of Physical Education, 1, 26-28.

Godik, M.A., Shanin, G.F., Shitikova, T.A. (1973). On the method of testing the physical condition of the children. Journal of Theory and Practice of Physical Education, 8, 32-35.

Godik, M.A. (1988). Sport metrology: the textbook for institutes of physical culture. M.: Physical Education and Sports, 192.

Zatsiorsky, V.M. (1979). Fundamentals of sports metrology. M.: Physical Education and Sports.

Lyakh, V.I. (1987). Questions diagnostic coordination abilities (based on foreign press). Journal of Theory and Practice of Physical Culture, 2, 56 .

Matveev, L.P. (1991). Theory and Methodology of Physical Education (general foundations of the theory and methodology of physical education, theoretical and methodological aspects of sports and professional applications forms of physical culture): a textbook for inst. of nat. culture. M.: Physical Education and Sports, 543.

Ozeretskiy, N. (1929). The method of mass valuation of motor children and adolescents. St. Petersburg, 178.

Ozolin, N.G. (1988). For a young colleague. M.: Physical Education and Sports, 228.

Ostanigrosh, N.M. (1984). Improving the teaching methods of control of physical development and physical fitness of students: diss. Kharkiv, 142.

Palagin, Y.S., Palagina, N.I., Polevshchikov, M.M. (2008). Physical training and its orientation Journal of Physical Education, Sport and Health: Sat. scientific. Art. Joshkar -Ola: Publishing house of the Mari state ped. Institute, 40-43.

Palagina, N.I., Polevshchikov, M.M. (2003). Standardized testing in the evaluation of motor abilities of students. Journal of Physical Education, Sport and Health: Sat. scientific. Art. Joshkar -Ola: Publishing house of the Mari state ped. Institute, 58-62.

Palagina, N. I. (2005). Optimization of physical preparation of students on the basis of the assessment of motor abilities: diss. ...Cand. ped. Sciences. Joshkar -Ola, 276.

Palagina, N. I. (2006). The problem of optimization of physical training of students on the basis of assessment of motor abilities. Journal of Physical Education, Sport and Health: Sat. scientific. Art. Joshkar -Ola: Publishing house of the Mari state ped . Institute, 8285.

Palagina, N.I., Blinova, M.L. (2010). The main directions of improvement of physical education students. Journal of actual problems of improving and adaptive physical education students; and Sat. scientific. Articles. Cheboksary Chuvashia. stateped. University Press, 181-186.

Palagina, N.I., Polevshchikov, M.M. (2013). On the issue of exercise in higher education. Journal of Physical Education, Sport and Health: Proceedings of the All-Russian scientific-practical conference. Yoshkar -Ola UnivMarg, 21-23.

Palagina, N.I., Polevshchikov, M.M. (2014). Metrological study of motor tests for assessing the level of physical fitness of students. Journal of Physical Education, Sport and Health: Proceedings of the All-Russian scientific-practical conference. Yoshkar -Ola Univ Marg, 91-96.

Palagina, N.I., Polevshchikov, M.M., Dorogova, Y.A., Blinova M.L., Zakamsky A.V., Shraga A.M., Loskutova E.A. (2015). Assessment of 
the Students' Physical Fitness Level and Metrological Justification of Motive. Review of European Studies. Vol. 7, No. 8, $137-146$. Polevshchikov, M.M., Palagina, N.I., Schraga, A.M. (2010). Methods of control in the process of improving physical education. Journal of Physical Education, Sport and Health: Proceedings of the All-Russian scientific-practical conference. Joshkar -Ola UnivMarg , 157-164.

Smirnov, Y.I. (1989). Key Features and performance sports readiness, Malakhovka: MOGIFK.

Smirnov, Y.I. (1989). Comprehensive assessment and control of sports readiness: a tutorial. - Malakhovka: MOGIFK.

Smirnov, Y.I., Polevshchikov, M.M. (2000). Sport metrology: Proc. for students. ped. universities. M .: publishing center "Academy".

Hasin, L.A., Rafalovich, A.B. (1995). Test to assess the physical fitness of students of institutes of physical culture magazine. Theory and Practice of Physical Education, 12, 43-48. 
